\title{
Un observatoire pour la montée de l'antisémitisme racial : les récits de pèlerinages en Terre sainte
}

Pierre Sauvage s. j.

\section{(2) OpenEdition \\ 1 Journals}

Édition électronique

URL : http://journals.openedition.org/cmc/1067

DOI : $10.4000 / \mathrm{cmc} .1067$

ISSN : 2684-3080

Éditeur

Fondation de la Mémoire Contemporaine

Édition imprimée

Date de publication : 1 janvier 2004

Pagination : 107-123

ISSN : 1377-1256

\section{Référence électronique}

Pierre Sauvage s. j., «Un observatoire pour la montée de l'antisémitisme racial : les récits de

pèlerinages en Terre sainte », Les Cahiers de la Mémoire Contemporaine [En ligne], 5 | 2004, mis en ligne le 01 novembre 2020, consulté le 30 janvier 2021. URL : http://journals.openedition.org/cmc/1067 ;

DOI : https://doi.org/10.4000/cmc.1067 


\title{
Un observatoire pour la montée de l'antisémitisme racial : les récits de pèlerinages en Terre sainte
}

\author{
Pierre Sauvage s. $\mathbf{j}$.
}

Cet article s'inscrit dans la foulée d'un ouvrage écrit en collaboration avec le professeur Guy Jucquois de l'Université catholique de Louvain, qui a comme titre: L'invention de l'antisémitisme racial. L'implication des catholiques français et belges (1850-2000)1. Dans ce livre est proposée l'hypothèse suivante: l'antisémitisme racial serait une attitude substitutive d'un antisémitisme religieux et ethnique (culturel). Je m'explique. Après leur émancipation, les Juifs quittant les ghettos se fondent dans la société occidentale et, par le fait même, ne sont plus reconnaissables par leur religion ou leurs habitudes culturelles. D'où naît une angoisse dans les sociétés occidentales, en majorité de tradition chrétienne, qui elles-mêmes souffrent d'un déficit identitaire, suite aux modifications des sociabilités causées, depuis la fin de l'Ancien Régime, notamment par l'urbanisation et l'industrialisation. Sont alors inventés des marqueurs indélébiles pour identifier les Juifs, la race et le sang, s'inspirant ainsi des matrices épistémiques évolutionnistes et biologiques qui prévalent à ce moment. Se met ainsi en place un processus de "diabolisation" du Juif dans lequel le déficit identitaire se projette sur l'autre qui en est rendu responsable. Ce phénomène explique pourquoi même des Juifs assimilés ont été envoyés dans les camps d'extermination.

L'hypothèse a été vérifiée par l'examen d'un ensemble de récits de pèlerinages qui couvrent la période de 1850 à 1940 . On a constaté que dans ces ouvrages les pèlerins non seulement parlent des Juifs rencontrés en Terre sainte, notamment au "Mur des Lamentations", mais qu'ils développent un discours sur l'ensemble de ce peuple. Le centre de l'ouvrage est donc consacré à l'analyse et à l'interprétation d'un corpus de ces récits de pèlerinages dans lesquels se perçoit la montée progres-

${ }^{1}$ Academia-Bruylant, coll. Sciences et Enjeux, Louvain-la-Neuve, 2001, 513 p. 
sive de l'antisémitisme racial. Pour bien comprendre tous les enjeux de cette analyse, il a paru indispensable de l'encadrer de deux parties. La première consiste à retracer sur la longue durée l'évolution de l'antisémitisme ; dans la seconde, qui s'intitule "Responsabilité et mémoire", les auteurs entendent répondre aux questions de fond soulevées par l'examen des récits et suivre l'évolution des relations entre Juifs et catholiques depuis la Shoah jusqu'aujourd'hui.

Le but de l'article n'est pas de résumer l'ensemble de la démarche suivie et tous les résultats obtenus, mais de montrer en quoi ces récits de pèlerinage sont un lieu de vérification privilégié de la montée de l'antisémitisme racial entre 1850 et 1940 . On se limitera ici à l'examen des récits écrits par des pèlerins belges. Avant d'expliquer en quoi ces écrits sont à la fois miroir et vecteur de cette mutation de l'antisémitisme, il est nécessaire, en guise de préliminaire, de donner des informations concernant, d'une part, l'organisation et l'esprit des pèlerinages et, d'autre part, les récits de pèlerinage.

\section{Les pèlerinages, organisation et esprit}

À partir du milieu du XIX $\mathrm{X}^{\mathrm{c}}$ siècle, les catholiques renouent avec la grande tradition des pèlerinages organisés vers la Terre sainte. Au fil du temps, cette organisation se développe et se perfectionne. Pour les catholiques, la Terre sainte est le pèlerinage par excellence, celui qui dépasse tous les autres : c'est là que Jésus-Christ a vécu, est mort et est ressuscité. C'est le berceau de leur religion. Dès 1853, un pèlerinage franco-belge, composé de quarante personnes, part pour la Palestine à l'initiative de la Société de Saint-Vincent de Paul de France, en réponse à un appel de la conférence de Jérusalem en faveur des pauvres chrétiens de cette ville ${ }^{2}$. L'CEuvre des Pèlerinages en Terre sainte est

2 Cl. Soetens, Les catholiques belges et le rapprochement avec les Églises d'Orient dans la seconde moitié du XIXème siècle dans Revue d'histoire ecclésiastique, t. 66, 1971, p. 86. A.-M. Portmans o.p. écrit que « l'Éuvre des pèlerinages en Terre sainte a été fondée en 1853, pour faciliter la visite des Lieux saints aux catholiques de tous les pays » (Pèlerinage en Terre sainte. Souvenirs et impressions, 1885, p. 288). Ces pèlerinages sont dirigés par un comité dénommé « (Euvre de la rue de Furstenberg ». Cfr. Rapporl du baron L. de Hodey sur l'Euvre des pèlerinages en Terre sainte dans Assemblée générale des catholiques en Belgique. Troisième session à Malines (2-7 septembre 1867), Bruxelles, 1867, p. 62. 
créée. À partir de cette date, sous la direction des Pères franciscains, qui sont gardiens des Lieux saints ${ }^{3}$, des caravanes se rendent en Terre sainte deux fois l'an, à Pâques et en août. Parmi les 618 participants qui gagnent la Palestine entre 1853 et 1870, on compte une bonne cinquantaine de Belges.

En 1882, les pèlerinages, qui s'essoufflaient depuis la guerre francoprussienne, prennent un nouvel élan : le père François Picard, un assomptionniste, met sur pied les « Pèlerinages populaires de Pénitence » qui, dès la première année, comptent plus d'un millier de pèlerins. Des Belges participent volontiers à ces caravanes. Ce n'est qu'en 1893 qu'ils organisent leur premier pèlerinage national ${ }^{4}$. Quelques années avant la Première Guerre mondiale, les franciscains de Gand créent le Commissariat de Terre sainte, qui se charge d'organiser les pèlerinages pour lesquels ils reçoivent l'approbation des évêques. En 1898, une autre organisation française voit le jour à l'initiative du chanoine Potard : elle porte le nom de Pèlerinage de Saint Louis. Interrompus durant la première guerre, les pèlerinages vers la Terre sainte reprendront de plus belle après le conflit.

L'esprit de pèlerinage peut se définir d'un mot : la croisade. Un rituel inspiré de celui des croisades précède l'embarquement des pèlerins dans le port de Marseille. Durant cette cérémonie, ils reçoivent de l'évêque du lieu la croix potencée qu'ils porteront ostensiblement sur leurs habits $^{5}$. Ce terme de croisade recouvre deux démarches différentes : sur la terre foulée par le Christ, les pèlerins accomplissent une démarche spirituelle pour nourrir leur foi mais, en même temps, ils entendent mar-

\footnotetext{
${ }^{3}$ En 1342, le pape fonde la Custodie de Terre sainte, une province essentiellement missionnaire de l'Ordre franciscain. Les franciscains reçoivent comme mission non seulement de conserver par la célébration des offices religieux, les droits des catholiques latins sur les grandes basiliques, mais aussi de récupérer les sanctuaires perdus et de relever les ruines des églises disparues. En outre, ils prennent en charge les pèlerins en les guidant et en les hébergeant. Jusqu'au milieu du XIX ${ }^{\mathrm{e}}$ siè- $^{-}$ cle, les franciscains sont les seuls religieux présents en Terre sainte : C. Aerts, o.f.m., Les franciscains au pays de Jésus, Gembloux, 1932, pp. 62-63.

${ }^{4}$ « Depuis mon premier voyage en Terre sainte, en 1884, je rêvais de refaire mon pèlerinage et de conduire aux Lieux saints une caravane belge. [...] Ce désir ardent de mon âme s'accomplit en 1893 : je fus chargé de la direction spirituelle de premier essai d'un pèlerinage belge aux Lieux saints » : A.-M. Portmans, En Égypte, Palestine et Grèce. Notes et impressions, Bruxelles-Paris, 1996, p. 1 .

5 J. Chelini et H. Branthomme, Les chemins de Dieu. Histoire des pèlerinages chrétiens des origines à nos jours, Paris, 1992, p. 317.
} 
quer leur territoire contre les « usurpateurs ». Ce n'est pas par hasard si la fondation de l'Euvre des Pèlerinages en Terre sainte est encouragée par le pape Pie IX. À ce sujet, un pèlerin belge écrit : « C'est une bonne œuvre que de visiter la Terre sainte. L'influence de la Russie y devient tellement prépondérante que si, de temps en temps, une caravane catholique ne vient pas par sa présence protester contre cet envahissement et montrer à ces peuples impressionnables que l'Europe songe encore à l'Orient, c'en est fait de notre crédit dans ces parages. $»^{6}$

Lorsque le père Picard crée les «Pèlerinages populaires de Pénitence », qui reçoivent l'appui du pape Léon XIII, l'esprit de croisade reste bien vivant, mais cette fois se produit un amalgame entre spiritualité et patriotisme. Puisque, depuis 1877, la Troisième République pratique une politique de sécularisation systématique et renoue avec la tradition du gallicanisme parlementaire, la démarche spirituelle des pèlerins s'accompagne de prières pour la conversion de la patrie ${ }^{7}$. L'esprit de croisade est à ce point ancré dans les mentalités des catholiques que, lorsque le chanoine Potard crée sa propre organisation, il l'intitule « Pèlerinages Saint-Louis ».

L'organisation belge ne fait pas exception pour unir spiritualité et patriotisme. Lors du pèlerinage de pénitence de 1884, les Belges invitent leurs compagnons à prier pour leur patrie, qui était à la veille d'élections législatives importantes pour le parti catholique. Le père Portmans o.p. justifie ainsi l'organisation de cette cérémonie : « N'estce pas en effet dans nos catholiques provinces, comme dans toute l'Europe, sous des appellations différentes, que se livre le grand combat entre Dieu et Satan, entre l'Église et l'athéisme, entre la cause de la vérité et de la justice et celle du mensonge et de la violence hypocrite ? $»^{8}$ Durant l'entre-deux-guerres, l'esprit de croisade reste dominant. Mais cette fois, il s'agit de faire valoir les droits de l'Église catholique face au Royaume-Uni qui, depuis 1922, a obtenu le mandat sur la Palestine. Il s'agit de faire pièce aux protestants. En 1932, pour fêter les cinquante ans de leur Euvre, les Assomptionnistes donnent plus de lustre au 75

\footnotetext{
${ }^{6}$ H. Ruelens, Journal d'un voyage en Terre sainte, Bruxelles, 1873, p. 6.

7 E. Lacoste, La vie du père François Picard, second supérieur général de la Congrégation des Augustins de l'Assomption, $1^{\text {er }}$ octobre 1831-1 $1^{\text {er }}$ avril 1903, Paris, 1932, pp. 295-296.

${ }^{8}$ Pèlerinage en Terre-Sainte. Souvenirs et impressions, Liège-Paris, 1885, pp. 256-257.
} 
pèlerinage. À cette occasion, ils éditent une brochure dans laquelle, en introduction, ils retracent l'histoire de leur œuvre et rendent un hommage vibrant au père François Picard, en qui ils saluent «le Pierre l'Ermite des croisades modernes aux Lieux saints $»$.

Dans un «Appel aux Belges », qui figure au début de la brochure éditée en 1930 par le Commissariat de Terre sainte, on peut lire : «Ici plus qu'ailleurs, les morts commandent, les aïeux invisibles mènent leurs descendants au tombeau du Christ. L'âme des trépassés dans ce qu'elle a de meilleur les anime, leur donne pensées et sentiments et fait revivre l'héroïsme et la bravoure de ces nombreux "soldats du Pape" comme Pirenne appelle nos pères, abandonnant leur famille et leur terre, pour aller en foule, riches et pauvres, vénérer le saint Sépulcre. Depuis sept siècles, les enfants de saint François continuent l'œuvre des Croisés, soutenus par les aumônes et les prières des fidèles du monde entier, qui, obéissant à la voix des papes, rappelle le vieux cri : "Dieu le veut!" " ${ }^{10}$ Trois ans plus tard, le père Beaufays abonde dans le même sens : il qualifie l'CEuvre des Pèlerinages en Terre sainte de « nouvelle croisade » et il rappelle la figure de « Godefroid de Bouillon, le premier roi de Palestine conquise par les Croisés ${ }^{11}$.

\section{Les récits de pèlerinages, succès et intérêt}

Le fait que les catholiques renouent avec la grande tradition des pèlerinages provoque une floraison de récits. Les pèlerins sont d'ailleurs encouragés à publier leurs souvenirs et leurs expériences. En 1864, des catholiques belges, réunis en assemblée, prennent comme résolution de " propager les écrits qui sont de nature à encourager les pèlerinages, notamment le Bulletin de l'CEuvre des pèlerinages en Terre sainte et le Bulletin de l'EEvre des Écoles d'Orient ${ }^{12}$. En 1882 , aux pèlerins qui viennent d'accomplir le Pèlerinage populaire de Pénitence, le pape Léon XIII s'adresse en ces termes : "Ces flammes de charité que vous avez ressenties aux lieux saints, efforcez-vous de les communiquer aux

\footnotetext{
${ }^{9}$ Année jubilaire. $75^{e}$ Pèlerinage national en Terre-sainte 10 août-17 septembre 1932, Paris, p. 5.

${ }^{10}$ Pèlerinage national belge aux Lieux saints, Commissariat de Terre sainte, Gand, 1930, p. 3.

11 I. Beaufays, Vers Jérusalem, Gembloux, 1933, pp. 10-11.

12 Assemblée générale des catholiques en Belgique. Seconde session 29 août-3 septembre 1864, op.cit., t. I, p. 466 .
} 
autres par la parole et par l'exemple. $»^{13}$ L'invitation est suivie d'effet. Un abbé, qui a publié ses souvenirs de pèlerinage, fait observer : «Depuis que ce conseil a été donné, les ouvrages se sont multipliés sous toutes les formes. $\aleph^{14}$

En lui-même, le récit de pèlerinage est une excellente pierre de touche pour se rendre compte de la mentalité des catholiques envers les Juifs. Comme dans tout récit de voyage, le pèlerin communique par la voie de l'écrit son expérience et ses impressions. Mais il ne se contente pas de décrire ce qu'il voit, il livre ses impressions, émet des avis et porte des jugements sur les hommes et sur les choses. Toutefois le pèlerin est un voyageur singulier ; il se déplace pour accomplir une démarche spirituelle et, dans son récit, il tente de restituer le mieux possible ce qu'il a vécu afin que le lecteur partage cette expérience et, au mieux, soit porté à s'embarquer pour la Terre sainte. Son récit est à la fois de purification et d'initiation.

C'est animé par cet esprit que le pèlerin livre ses impressions et ses jugements sur les Juifs qu'il a nécessairement rencontrés lors de son périple. Entre 1880 et 1940 , les occasions pour les pèlerins de rencontrer les Juifs se multiplient. À partir de 1882, un certain nombre de Juifs fuyant les pogroms en Europe centrale et orientale trouvent refuge en Palestine. C'est le début des grandes aliyot (montées) qui s'amplifieront au cours des années. En 1896, Théodore Herzl, avec la création du sionisme politique, donne une impulsion décisive à ce mouvement de retour. En 1917, la déclaration Balfour accélère définitivement le processus.

Avec ces récits, on dispose donc d'un véritable "laboratoire" où se manifestent insensiblement, sous forme d'un adjectif, d'une caractéristique, d'une appréciation morale ou physique, tous les traits qui, à terme, tracent le portrait du Juif tel qu'il est dépeint par l'antisémitisme racial. L'intérêt de ces récits réside précisément dans le fait qu'ils n'ont pas pour objet premier et explicite de porter un jugement sur les Juifs et ainsi d'inculquer au lecteur des modes de pensée antisémite. Ce

\footnotetext{
${ }^{13}$ Phrase citée par V-A. Mourot, La Terre sainte et le pèlerinage de pénitence de 1882. Impressions el souvenirs, Mirecourt, 1882, p. 16.

${ }^{14}$ L. Alazard, En Terre sainte. Monographie des saints Lieux ; Souvenirs de pèlerinage, Rodez, 1895 , p. 7 .
} 
n'est pas l'antisémitisme qui rassemble les pèlerins, mais bien le fait d'être de bons catholiques soucieux de faire une forte expérience spirituelle. C'est donc incidemment que ces pèlerins décrivent les Juifs et communiquent leurs impressions et leurs jugements à leur sujet. C'est un témoignage in obliquo.

Comme toute source historique, le récit de pèlerinage doit être soumis à la critique. Si la plupart des auteurs affirment que leur désir est de transmettre leurs expériences et leurs impressions, il ne faudrait pas en conclure qu'ils fournissent un récit spontané, dans le sens où les auteurs se contentent de communiquer simplement tout ce qu'ils ont vécu et vu pendant leur séjour. D'une part, le récit comporte une "mise en scène", ne fût-ce que par le découpage en chapitres, qui est toujours une opération artificielle. C'est donc un récit construit. D'autre part, l'auteur, même s'il prétend le contraire, ne transmet jamais la totalité de son expérience. Il sélectionne nécessairement ses matériaux et, pour opérer ce tri, il utilise une série de critères qui lui sont propres.

Il importe également de tenir compte du fait que les auteurs de ces récits peuvent s'inspirer des récits et des guides de Palestine qui sont sur le marché. En ce qui concerne l'objet de notre recherche, nous en avons un bel exemple. Dans leur jugement sur les Juifs, les pèlerins ont été influencés par Liévin de Hamme, un franciscain d'origine belge établi en Palestine depuis $1859^{15}$. Ce religieux exerce deux activités complémentaires. D'une part, il guide les pèlerins : il est le délégué du Custode pour diriger les caravanes françaises en Terre sainte ; à partir de 1882, il est choisi par les Assomptionnistes pour les seconder lors des Pèlerinages populaires de Pénitence; en 1893, il dirige le premier pèlerinage national belge. Il accomplit cette tâche jusqu'en 1897, un an avant sa mort. L'homme est une figure connue et ses services sont appréciés : un pèlerin belge le qualifie « d'ange gardien et de bon génie du

\footnotetext{
15 Pour la biographie de ce religieux, voir A. Ceyssens, o.f.m, Le frère Liêvin de Hamme. Frère mineur de la province de Saint-Joseph de Belgique. Guide officiel des Pèlerins en Terre-Sainle - membre de la Société biblique de Rome - Officier d'Académie de France - Chevalier de l'ordre des Saints Maurice et Lazare, d'Italie, Précédé d'un aperçu historique sur les Franciscains en Terre-Sainte, Gand, 1903. On trouvera une présentation plus complète de la personnalité et de l'activité de ce religieux franciscain dans L'invention de l'antisémitisme racial. L'implication des catholiques français et belges, op.cit., pp. 251-264.
} 
pèlerinage $\aleph^{16}$. D'autre part, le franciscain est l'auteur du premier guide de la Palestine destiné aux pèlerins d'expression française. Publié en 1869, le petit ouvrage s'intitule: Guide indicateur des sanctuaires et lieux historiques de la Terre-Sainte. L'opuscule connaît un tel succès qu'il est l'objet de trois éditions (en 1876, en 1887 et en 1897), dont chacune fera l'objet d'une mise à jour. Son succès atteste son influence sur des générations de pèlerins. Ils y puisent non seulement des conseils pour l'itinéraire à suivre et des informations historiques et archéologiques dignes de confiance, mais également ils y trouvent des interprétations des données religieuses et culturelles. Or, dans son guide, le franciscain parle des Juifs à une seule occasion, lors de la visite au "Mur des Lamentations", qu'il conseille de visiter le vendredi après-midi au moment où les Juifs sont assez nombreux ${ }^{17}$. On peut constater que, dans son commentaire, le franciscain développe la plupart des stéréotypes religieux concernant les Juifs. En recommandant cette visite, le religieux poursuit un but apologétique : prouver la divinité du christianisme en montrant que les prophéties sont accomplies.

\section{Impressions et jugements de pèlerins belges}

Le corpus de récits qui a été étudié comporte quatre-vingt-quatre récits de pèlerinages, qui couvrent une période qui s'étend de 1854 à 1936. Parmi ceux-ci, quinze récits sont écrits par des Belges ${ }^{18}$. À l'exception d'un seul, tous (soit neuf) sont des clercs qui en majorité font partie du clergé séculier - on sait que six sont curés de paroisse et deux assurent la fonction de professeurs : l'abbé Léon Courtois et le chanoine Paul Halflants. Parmi les religieux, on compte un dominicain, le père Portmans, deux franciscains, le père Beaufays et le frère Meunier, et un bénédictin qui a le titre d'évêque, Mgr Henri-Laurent Janssens.

16 A.M. Portmans, Pèlerinage en Terre-Sainte. Souvenirs el impressions, Liège-Paris, 1885, p. 22.

17 Guide indicateur des sanctuaires et des lieux historiques de la Terre-Sainte, Louvain, 1876, p. 319.

18 On trouvera la liste complète de ces ouvrages en annexe. 


\section{Permanence des stéréotypes religieux}

Dans l'examen de ces récits, on retrouve les stéréotypes religieux qu'au cours du temps le christianisme a élaborés à l'égard des Juifs. Ils se rencontrent principalement lors de la visite au "Mur des Lamentations" prévue lors des pèlerinages organisés et recommandée par le guide du frère Liévin de Hamme. La visite au Mur constitue un moment privilégié où les pèlerins expriment ce qu'ils ressentent non seulement envers ces quelques Juifs qu'ils voient, mais envers l'ensemble de ce peuple.

Pour qualifier la cérémonie des Juifs qui « pleurent » devant le Mur, la majorité des pèlerins emploient le terme « spectacle », ce qui implique une volonté de prendre distance et de ne pas se mêler de cette cérémonie. Par exemple, le chanoine Hoornaert commente la scène de cette manière: «Aujourd'hui, Vendredi-Saint, allons les [les Juifs] trouver chez eux ; ils nous donneront le spectacle traditionnel de leurs lamentations devant le Mur des Pleurs. ${ }^{19}$

Quels sont ces stéréotypes ? Tout d'abord, les pèlerins véhiculent le thème traditionnel de l'expiation du déicide et de l'accomplissement des Écritures. Le même chanoine fait le commentaire suivant: «La mission de juifs est de représenter authentiquement des ancêtres déicides. » C'est ce qui explique qu'au Mur « ils ont l'attitude des condamnés qui expient leurs crimes $»^{20}$. Un autre pèlerin écrit : «Il m'a toujours paru que dans la lecture des prophètes, lecture parfois difficile, c'est la prédiction des destinées d'Israël, de sa chute profonde et irrémédiable, de sa dispersion dans le monde qui constitue l'essentiel. » Et il ajoute : «Ne voyons nous pas de nos yeux l'accomplissement de cette prédiction ? " ${ }^{21}$ Après avoir visité le quartier juif de Jérusalem, un pèlerin fait les réflexions suivantes : «Pauvres gens! Ils vivent au milieu des ruines prédites par leurs prophètes; ils palpent pour ainsi parler l'accomplissement des prophéties ; ils portent les malédictions qu'ils ont appelées sur eux devant le prétoire de Pilate; depuis dix-huit siè-

19 Terre promise el Palestine moderne, Courtrai, 1914, p. 195.

20 Ibid., pp. 194 et 197.

${ }^{21}$ Éd. De Gryse, Voyage en Orient, Grèce, Palestine, Égypte, Ypres, 1909, pp. 172-173. 
cles, ils voient les œuvres du Messie resplendir dans le monde; et cependant ils s'obstinent dans un incroyable entêtement. Leurs yeux sont fermés à la lumière apportée par le Christ, lumière plus éblouissante que le soleil en son midi; leurs cœurs orgueilleux se raidissent contre toutes les tentatives qu'on fait journellement pour les ramener à la vérité. Triste aveuglement! Obstination plus triste encore ! Déicide des juifs, voilà ta punition! Oh que le Ciel a finalement exaucé le terrible souhait des meurtriers de Jésus: "Que son sang retombe sur nous et sur nos enfants!" " ${ }^{22}$ On retrouve ensuite l'insistance sur la vanité des prières juives. À ce propos, quelques ecclésiastiques accompagnent leurs réflexions d'une certaine commisération : « Les pauvres gens! [...] Ils s'adressent au mur comme si celui-ci pouvait les comprendre. ${ }^{23} \mathrm{Un}$ autre insiste sur la sincérité de leur démarche : «Tous [...] laissent tomber de vraies larmes qui arrosent la pierre insensible. $»^{24} \mathrm{Un}$ autre pèlerin insère ses observations dans un contexte historique : "Depuis que l'Islam leur a défendu, sous peine de mort, de pleurer la destruction de leur royaume près de la grande mosquée, ce mur est le refuge de leur douleur. Ils en ont maintenant le culte, et lui offrent, comme à une divinité muette, le sacrifice perpétuel de leurs gestes navrés et de leurs larmes [...]. D'autres, moins passifs, après avoir essuyé leurs larmes contre le granit insensible, comme pour lui reprocher son inaction et réclamer l'accomplissement des textes qu'ils profèrent... $\gg^{25}$ Enfin, on retrouve un appel à la conversion. Un abbé rappelle le sens de la prière liturgique du Vendredi saint : "L'Église, chose admirable, a une compassion spéciale pour "les Juifs perfides". Précisément dans l'office du jour, elle impose une prière touchante pour eux et demande à Dieu, qui n'a pas exclu les déicides de sa miséricorde, la grâce qui les arrachera à leurs ténèbres. ${ }^{26}$ Se fondant sur les paroles de l'apôtre Paul, un autre auteur présente la conversion des Juifs comme une certitude à la fin des temps: "L'espérance que les juifs s'obstinent à nourrir encore confirme la prédiction de saint Paul. Ces espérances se réaliseront

\footnotetext{
22 A. Ruelens, Journal d'un pèlerin en Terre sainte, Malines, 1873, p. 212.

23 Ibid., p. 199.

${ }^{24}$ L. Courtois, Jérusalem et les Lieux saints de Palestine. Impressions et souvenirs, Namur, 1901, p. 57.

${ }^{25}$ H. Hoornaert, Terre promise et Palestine moderne. Courtrai, 1914. p. 196.

26 Ibid., p. 199.
} 
quand le peuple juif finira par reconnaître que Jésus est le seul Messie qu'ont attendu leurs pères et que préfigurait le temple de Salomon. „27

Lorsque les pèlerins élargissent leurs réflexions à l'ensemble du peuple juif, apparaissent d'autres stéréotypes traditionnels. Ce peuple qui a traversé les siècles est, malgré lui, porteur d'une double mission : témoigner de la toute-puissance de Dieu et endurer les persécutions et les malheurs, comme conséquences du déicide. Comme Caïn, il est condamné à l'errance. À ce propos un pèlerin écrit en 1896 : « Le peuple juif porte au front le stigmate de la justice divine qui le poursuit dans tous les lieux de son exil, parmi toutes les nations, même en terre musulmane. ${ }^{28}$

\section{Face au sionisme}

Le stéréotype traditionnel de l'errance est ébranlé par le retour important des Juifs en Palestine amorcé à la suite des pogroms en Russie et amplifié lors de la création du sionisme politique par Herzl et surtout par la déclaration Balfour. Durant l'entre-deux-guerres, trois pèlerins belges émettent des opinions défavorables face au sionisme. Dès 1921, Mgr Janssens écrit que «la nouvelle ville juive prend chaque jour plus d'extension du côté Nord-Ouest de Jérusalem. C'est de ce côté-là, sans doute, que se développera la ville, si les tristes projets sionistes, caressés en Angleterre et aux États-Unis, doivent se réaliser. Quod Deus advertat ! ${ }^{29}$ Quatre ans plus tard, le chanoine Paul Halflants est encore plus explicite. Il consacre un des appendices de son ouvrage à mettre en lumière ce qu'il appelle « Le danger du sionisme ». Selon lui, « c'est l'idée nationaliste, beaucoup plus que l'utopie de la reconstruction du Temple et du rétablissement de la loi mosaïque, qui hante les cerveaux des ces "Amis de Sion", dont une bonne partie est gagnée au bolchevisme $\aleph^{30}$. C'est pourquoi, adressant une sérieuse mise en garde envers l'Angleterre qui a obtenu le mandat sur la Palestine, il écrit : «Certes, il n'est pas facile de gouverner la Palestine, où les plus étran-

${ }^{27}$ H.-L. Janssens, Au pays du Messie, Paris-Bruxelles, 1921, p. 359.

28 A.-M. Portmans, Pèlerinage en Terre sainte. Souvenirs et impressions, Liège, 1896, p. 128.

${ }^{29}$ Au pays du Messie, 1921, Paris-Bruelles, p. 118.

${ }^{30}$ P. Halflants, Autour de la Méditerranée. Lettres d'un pèlerin de Jérusalem. Paris-Bruxelles, 1925, p. 173. 
ges fanatismes vivent côte à côte, sans s'amalgamer, toujours prêts à en venir aux mains! Mais le projet d'y établir un État juif, sous prétexte de rendre aux Israélites la terre de leurs ancêtres est, bien même du point de vue purement humain et politique, l'une des plus folles qu'ait pu inspirer le nationalisme. Sans même parler du droit des catholiques, il faut, pour aboutir à une conception pareille, bien peu tenir compte du fanatisme des sectes chrétiennes et des musulmans : se figure-t-on que le Saint Sépulcre et la Mosquée d'Omar seront ainsi abandonnés aux juifs sans coup férir ? Que de tragédies sanglantes l'Angleterre prépare là-bas, si elle persiste à protéger un mouvement sioniste, qui ne peut devenir qu'un nouvel et terrible élément de discorde! $\|^{31}$

En 1931, un religieux trace un portait peu flatteur des sionistes: « La langue hébraïque, des prophètes et des écrivains sacrés, renaît et avec elle la poussée messianique, plus impérieuse que jamais, car ces sionistes, habillés en scouts comme les ouvriers de nos usines, sont convaincus qu'Israël est un peuple élu, le premier des peuples, que son avenir est radieux. Las d'implorer le ciel et d'attendre sa rosée, ils se tournent farouchement vers la terre et veulent en faire jaillir le paradis perdu. Ils rêvent de richesses, de bien-être, de repos, grâce au collectivisme intégral. Comme les Sadducéens d'autrefois, ils ne croient ni aux esprits, ni à la résurrection, mais s'arrangent avec les maîtres du pays, pour hâter l'accomplissement de leurs vœux et asseoir leur domination universelle... C'est pourquoi "le peuple de la terre", l'arabe musulman, les hait et les gens pieux d'Israël leur lancent l'anathème : “À moins que le Seigneur bâtisse la demeure, c'est en vain que travaillent les maçons!"” ${ }^{32}$

\section{Une attention croissante au physique des Juifs}

Dès la seconde moitié du $\mathrm{XIX}^{\mathrm{e}}$ siècle, à côté des stéréotypes religieux, apparaissent dans les récits des observations qui touchent le physique des Juifs, principalement celui des hommes adultes. Ayant séjourné à Tibériade, l'abbé Ruelens fait cette constatation : « La plu-

31 Ibid., pp. 177-178.

32 I. Beaufays, Vers Jérusalem, Gembloux, 1931, p. 46. 
part des habitants que nous rencontrons sont des juifs, au nez caracté-

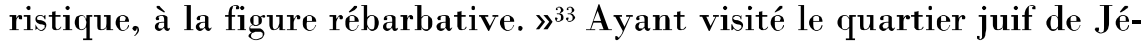
rusalem, il fait observer : « Les juifs sont là entassés au nombre d'environ 7.000 entre le mont Sion et le temple, dans de misérables masures qu'on n'oserait décorer du nom de maisons, vivant Dieu sait comment et de quoi. ${ }^{34}$ Tout se passe comme si le "physique repoussant" des Juifs contaminait leur environnement.

À partir de la fin du siècle, une évolution se dessine : les remarques se multiplient et prennent davantage et globalement un caractère négatif. C'est l'époque où l'antisémitisme racial arrive à maturité. À propos des Juifs de Jérusalem, un auteur écrit : «On reconnaît les juifs à leur air sordide et blême, à leurs cheveux roulés en tire-bouchons sur les tempes. $\aleph^{35}$ L'impression du père Portmans, qui a visité le quartier juif, est plus nuancée : «Au milieu de cette foule puante, graisseuse, loqueteuse, à côté de ces enfants à la figure livide et anémiée surgissent des types beaux et impressionnants. Nulle part on ne voit un tel choix de portraits d'Abraham, de Jacob... et aussi de Judas. Voyez ce vieillard à la barbe blanche : quelle intensité d'expression, quel rayonnement de l'âme, quelle fatigue de la vie, et néanmoins quels regards profonds et scrutateurs! N'est-ce pas le vieux juif de Rembrandt? Oui, hormis les affreuses mèches sur leurs tempes, qui sont d'importation polonaise. Superbes aussi quelques têtes de femmes et de jeunes filles, vraies Rachel, Judith, Esther. Et aussi quelle gravité, quel air viril, quelle force et quelle douceur chez ces jeunes gens qui n'ont pas, eux, les cheveux en papillotes et qui font rêver du Christ. Ah ! je comprends pourquoi mon ami, le peintre, aimait à errer dans ce quartier aux heures matinales: il y trouvait des modèles pour ses tableaux, et il s'inspirait au contact de ces types expressifs, primitifs, qui sentent la race. $\aleph^{36}$

33 Journal d'un pèlerin en Terre sainte, Malines, op.cil., p. 375.

34 J. Ruelens, Journal d'un pèlerin en Terre sainte. Malines, 1873.

35 J. Goedert, Un pèlerinage à Jérusalem, Rome et Lourdes, Namur, 1904, p. 42. L'auteur fait allusion aux papillotes, «péot » (littéralement « coins » en hébreu) portées par certains Juifs : « Longues boucles de cheveux que certains juifs ultra-orthodoxes se laissent pousser sur les tempes, conformément à l'injonction biblique : "Vous ne couperez pas en rond les coins de votre tête" (Lévilique, XIX, 27) » (A. Unterman, Diclionnaire du judaisme. Histoire, mythes el tradilions, Paris, 1996, p. 226)

36 Pèlerinage en Terre sainte. Souvenirs et impressions, Liège-Paris, 1885, p. 122. 
En dehors de Jérusalem, des pèlerins ont l'occasion de se rendre en Galilée, où ils visitent la petite ville de Tibériade. Leur jugement n'est pas très favorable. « La Tibériade moderne est une misérable bourgade de près de quatre mille habitants, la plupart juifs. On se fait difficilement une idée de la malpropreté de ce trou, dont les Israélites avaient rêvé de faire une seconde Jérusalem ", écrit le Père Portmans ${ }^{37}$. Un autre pèlerin écrit : «La population est d'environ 6.000 âmes, dont 20 Latins, 250 Grec-Unis, 600 musulmans ; tout le reste est juif, et c'est sans doute à cela qu'on doit attribuer la malpropreté de la ville. Ces gens ont un plus grand soin de leur bourse que de leur personne, de leur maison et de leur ville. $\rrbracket^{38}$ Le lien entre la présence de Juifs et la saleté de la ville est encore clairement souligné par deux auteurs : « Tibériade est un vrai fief des juifs. Les $2 / 3$ de la population sont des descendants d'Abraham. Tibériade est la ville le plus sale que nous ayons rencontrée dans tout l'Orient », affirme l'abbé Goedert ${ }^{39}$. Le chanoine Hoornaert ajoute encore d'autres traits dépréciatifs : « L'étalage désuet du dehors promet quelques chose; l'intérieur ne donne rien et tient du lazaret ou de l'asile de vagabonds. Ni activité, ni vie. Au long de ruelles malpropres, des masures croulantes se soutiennent l'une l'autre, de guingois la plupart, et cela forme une ville sur une bande de terre assez étroite entre le lac et la montagne. Les juifs aiment ce refuge ; il flatte leurs traditions, car après la ruine de Jérusalem, Tibériade s'éleva à la dignité d'une ville sainte et devint un centre d'enseignement rabbinique réputé. Maintenant des juifs, surtout des Polonais, sont envoyés ici par des coreligionnaires qui leur fournissent le nécessaire. Par reconnaissance, ils parlent allemand [...]. Aucune industrie n'est apparente. $»^{40}$

Au terme de cette analyse, on peut constater que dans tous ces témoignages de pèlerins concernant les Juifs, l'attention aux traits physiques se conjugue progressivement non seulement avec les stéréotypes religieux traditionnels, mais également avec des appréciations négatives relatives aussi bien à l'habillement, à la toilette, à la coiffure et à

37 Ibid., p. 43.

${ }^{38}$ A. De Lancker, Lettre sur la Terre-Sainte ou récit d'un voyage en Égypte, en Palestine et dans le Liban, 1890, p. 113.

${ }^{39}$ Un pèlerinage à Jérusalem, Arlon, 1904, p. 125.

${ }^{40}$ Terre promise et Palestine moderne, Courtrai, 1914, p. 91. 
l'hygiène. On peut affirmer que l'antisémitisme racial n'élimine pas les autres formes d'antisémitisme, qu'il soit religieux ou ethnique; il les complète en quelque sorte et les compense si ces dernières risquaient de disparaître. Il faut néanmoins reconnaître que, dans les récits que nous avons étudiés, la percée de l'antisémitisme racial ne se perçoit pas de manière aussi évidente que dans les récits de pèlerins français. On peut constater aisément cette différence dans l'ouvrage cité au début de cet article. Cela peut s'expliquer de différentes manières. Une raison superficielle : dans le corpus que nous avons constitué, le nombre des récits écrits par des pèlerins belges est peu élevé par rapport à celui écrits par les français (15/84). Les témoignages sur les Juifs sont donc nécessairement moins abondants. Une autre explication, plus profonde, tient sans doute à la mentalité belge. Habitués depuis longtemps au "compromis", les Belges, d'une manière générale, n'ont pas tendance, comme dans d'autres pays occidentaux, notamment la France, à radicaliser une situation et donc sont plutôt portés à être plus discrets dans la manifestation de l'antisémitisme racial, même si celui-ci est bien présent $^{11}$. Ceci peut en partie se comprendre du fait que, à cette époque, en Belgique, la présence juive est beaucoup moins nombreuse qu'en France.

\section{En guise de conclusion}

Malgré les limites du travail, on a pu se rendre compte combien les récits de pèlerinage, qui appartiennent à la littérature de voyage, constituent un observatoire de choix pour suivre l'évolution des mentalités à propos des Juifs. Les résultats ainsi obtenus complètent ou confirment avantageusement ceux recueillis lors de l'examen d'autres types de sources.

Je terminerai par une invitation aux chercheurs : exploiter davantage ce champ de recherche que constituent les récits de voyage en privilégiant deux directions. D'une part, élargir le corpus des écrits des pèlerins belges de cette époque et le compléter par une étude des récits

\footnotetext{
41 On pourra trouver un développement plus ample de cette explication dans L'invention de l'antisémitisme racial. L'implication des catholiques français et belges. notamment dans le chapitre 10 , consacré aux "Spécificités belges", pp. 157-192.
} 
écrits par des simples voyageurs de nationalité belge, afin de comparer leur perception du monde juif en Palestine avec celle des pèlerins. D'autre part, ouvrir d'autres chantiers qui concernent le monde juif en Palestine : par exemple étudier le sionisme (une amorce a été donnée dans cet article) et les relations entre Juifs sionistes et non sionistes et Palestiniens. Je suis convaincu que ces études apporteraient des éclairages dignes d'intérêt.

\section{Annexe : récits de pèlerinages étudiés}

- Ignace Beaufays, o.f.m., Vers Jérusalem, Gembloux, Duculot, 1931.

- Léon Courtois, Abbé. Professeur au Collège Saint-Joseph à Virton, Jérusalem et les Lieux saints de Palestine. Impressions et souvenirs, Namur, Godenne, 1901.

- Éd. De Gryse, Abbé. Curé-doyen de sainte Catherine à Cuerne, diocèse de Bruges, Lettre sur la Terre sainte ou récit d'un voyage en Egypte, en Palestine et dans le Liban, traduit du flamand avec l'autorisation de l'auteur, Braine-le-Comte, Imprimerie Lelong, 1890.

- J. Goedert, Abbé. Curé à Fouches, Un pèlerinage à Jérusalem, Rome et Lourdes, nouvelle édition revue et augmentée, Namur, Libraire Picard-Balon, 1904.

- Id., Ancien curé de Fouches. Retraité à Arlon, Un pèlerinage à Jérusalem, Arlon, Imprimerie de la presse luxembourgeoise, 1920.

- Paul Halflants, Chanoine. Professeur à la faculté de Philosophie et Lettres de l'Institut Saint-Louis de Bruxelles, Autour de la Méditerranée. Lettres d'un pèlerin de Jérusalem, Paris-Bruxelles, DDB, 1925.

- Hector Hoornaert, Chanoine, Terre promise et Palestine moderne, Courtrai, Vermaut, 1914.

- Edmond Jamart, Curé de Baulers, Notes au jour le jour d'un voyage en Terre sainte offertes à ses paroissiens, Nivelles, Havaux-Houdart, 1915.

- Henri-Laurent Janssens, o.s.b., Évêque titulaire de Bethsaïde, $A u$ pays du Messie, Paris-Bruxelles, DDB, 1921. 
- Fulgence Meunier, o.f.m., À Jérusalem par la Croatie, les Balkans, l'Asie Mineure et la Syrie, Liège, 1929.

- Henri Nicodéme, Abbé, Le pèlerinage en Terre sainte, Luttre, Office international d'Éditions, Alfred Balsacq, 1928.

- A.-M. Portmans, o.p., Pèlerinage en Terre sainte. Souvenirs et impressions, Liège-Paris, H. Dessain, 1885.

- Id., En Égypte. Palestine et Grèce. Notes et impressions, Société belge de Librairie, Bruxelles-Paris, Victor Lecoffre, 1896.

- Auguste Ruelens, Curé de Héverlée-lez-Louvain, Journal d'un pèlerin en Terre sainte, Malines, 1873.

- Firmin Van den Bosch, Le long de ma route. Égypte-Palestine-Grèce, Bruxelles-Paris, Giraudon, 1924. 